\title{
PDFgetN3: atomic pair distribution functions from neutron powder diffraction data using ad hoc corrections
}

\author{
Pavol Juhás, Jaap N. Louwen, Lambert van Eijck, Eelco T. C. Vogt and \\ Simon J. L. Billinge
}

J. Appl. Cryst. (2018). 51, 1492-1497

\section{IUCr Journals CRYSTALLOGRAPHY JOURNALS ONLINE \\ Copyright (C) International Union of Crystallography \\ Author(s) of this paper may load this reprint on their own web site or institutional repository provided that this cover page is retained. Republication of this article or its storage in electronic databases other than as specified above is not permitted without prior permission in writing from the IUCr. \\ For further information see http://journals.iucr.org/services/authorrights.html}




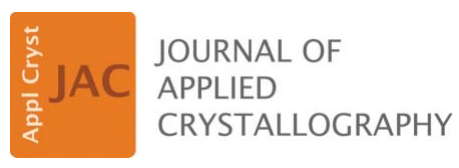

ISSN 1600-5767

Received 7 May 2018

Accepted 11 July 2018

Edited by Th. Proffen, Oak Ridge National Laboratory, USA

Keywords: neutron diffraction; pair distribution functions; constant-wavelength neutron

instruments; computer programs.

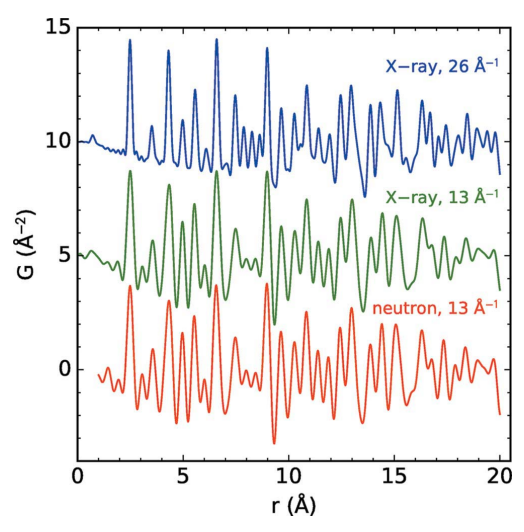

(C) 2018 International Union of Crystallography

\section{PDFgetN3: atomic pair distribution functions from neutron powder diffraction data using ad hoc corrections}

\author{
Pavol Juhás, ${ }^{\mathrm{a}}$ Jaap N. Louwen, ${ }^{\mathrm{b}}$ Lambert van Eijck, ${ }^{\mathrm{c}}$ Eelco T. C. Vogt ${ }^{\mathrm{b}, \mathrm{d}}$ and \\ Simon J. L. Billinge ${ }^{\mathrm{e}, \mathrm{f}_{*}}$
}

\begin{abstract}
${ }^{a}$ Computational Science Initiative, Brookhaven National Laboratory, Upton, NY 11973, USA, ${ }^{\mathbf{b}}$ Albemarle Catalysts Company BV, PO BOX 37650, 1030 BE Amsterdam, The Netherlands, ' Department NPM2/RST, Faculty of Applied Sciences, Delft University of Technology, Delft, The Netherlands, 'Debye Institute of Nanomaterials Science, Utrecht University, Universiteitsweg 99, 3584 CG Utrecht, The Netherlands, ${ }^{\mathbf{e}}$ Department of Applied Physics and Applied Mathematics, Columbia University, New York, NY 10027, USA, and 'Condensed Matter Physics and Materials Science Department, Brookhaven National Laboratory, Upton, NY 11973, USA. *Correspondence e-mail: sb2896@columbia.edu
\end{abstract}

PDFgetN3, a new software tool for the extraction of pair distribution functions (PDFs) from neutron powder diffraction intensity data, is described. Its use is demonstrated with constant-wavelength neutron data measured at the new powder diffractometer PEARL at the Delft University of Technology. PDFgetN3 uses an ad hoc data collection protocol similar to PDFget X3. The quality of the resulting PDFs is assessed by structure refinement and by comparison with established results from synchrotron X-ray scattering.

\section{Introduction}

The analysis of total scattering and pair distribution functions (PDFs) has become increasingly popular in the field of nanomaterial structural analysis (Egami \& Billinge, 2012). Nanomaterials often do not display sufficient long-range order to allow traditional structure analysis by diffraction measurements (Billinge, 2010). A number of beamlines for both X-rays and neutrons have been optimized for total scattering and PDF analysis (Egami \& Billinge, 2012). Raw data from these instruments need to be processed by correcting for experimental artifacts and normalizing. Several software tools are available to extract the PDF from the scattering patterns, such as GudrunX (Soper \& Barney, 2011; http://www.isis.stfc.ac.uk/instruments/sandals/data-analysis/ gudrun8864.html), RAD (Petkov, 1989) and PDFgetX2 (Qiu et al., 2004) for X-rays, and GudrunN (http://www.isis. stfc.ac.uk/instruments/sandals/data-analysis/gudrun8864.html) and PDFgetN (Peterson et al., 2000) for neutrons. These programs all use elaborate correction algorithms that require a multitude of complicated user inputs. The program PDFgetX3 (Juhás et al., 2013), which uses an ad hoc correction algorithm, was developed to overcome this problem. It was found to produce PDFs of comparable quality to those obtained using PDFgetX2, which implements all the corrections, but with much less user input and much more quickly.

All the tools described above are for X-ray or time-of-flight neutron analysis. There are few options for obtaining the PDF from constant-wavelength neutron data. The existing codes are available as instrument-specific utilities (Fischer, 2016; Howe \& McGreevy, 1996), which are not easy to obtain and use elsewhere. The new software PDFgetN3 introduced here 
provides the PDF extraction capability in a freely available, user-friendly, software program. While developed for constant-wavelength intensity versus $2 \theta$ data, the program has also been successfully tested with intensity versus $Q$ data acquired from a time-of-flight instrument.

The PDF in the $G(r)$ form is calculated by Fourier transformation of the reduced structure function (also called 'interference function') $F(Q)$ (Egami \& Billinge, 2012):

$$
G(r)=(2 / \pi) \int_{Q_{\min }}^{Q_{\max }} F(Q) \sin Q r \mathrm{~d} Q,
$$

where $F(Q)$ is expressed in terms of the structure function, $S(Q)$, as

$$
F(Q)=Q[S(Q)-1]
$$

and $S(Q)$ derives from the coherent scattering intensity normalized per atom $I_{\mathrm{c}}(Q)$. For the case of X-ray scattering, $S(Q)$ is given by

$$
S(Q)=\frac{I_{\mathrm{c}}(Q)-\left\langle f_{i}(Q)^{2}\right\rangle+\left\langle f_{i}(Q)\right\rangle^{2}}{\left\langle f_{i}(Q)\right\rangle^{2}},
$$

where $f_{i}(Q)$ is the $Q$-dependent X-ray scattering factor of atom $i$, and the averaging is done over all atoms. In general, the coherent intensity $I_{\mathrm{c}}$ is extracted from the measured intensity $I_{\mathrm{m}}$ by correcting for a number of contributions to the intensity that multiply or add to the coherent signal:

$$
I_{\mathrm{m}}(Q)=\alpha(Q) I_{\mathrm{c}}(Q)+\beta(Q) .
$$

Incoherent Compton and background scattering contribute to the additive corrections, whereas self-absorption and polarization contribute to the multiplicative corrections.

Billinge \& Farrow (2013) showed that it is possible to get a sufficiently accurate estimate of $\alpha(Q)$ and $\beta(Q)$ for X-ray scattering by applying an ad hoc approach. Although the method was developed for X-ray scattering it also works for neutron scattering, by applying a small change to the structure function $S(Q)$ :

$$
S(Q)=\frac{I_{\mathrm{c}}(Q)-\left\langle b_{i}^{2}\right\rangle+\left\langle b_{i}\right\rangle^{2}}{\left\langle b_{i}\right\rangle^{2}},
$$

where $b_{i}$ is the ( $Q$-independent) coherent neutron scattering length of atom $i$, and the angle brackets indicate an average over all atoms in the sample. Finally, the adjusted $S(Q)$ feeds into equations (2) and (1) to allow calculation of the PDF.

\subsection{PEARL diffractometer}

The neutron powder diffractometer PEARL at TU Delft is a constant-wavelength $(\mathrm{CW})$ diffractometer. The wavelength of the neutrons that impinge on the sample in such $\mathrm{CW}$ instruments is selected from a polychromatic neutron beam by a monochromator. Upstream of the monochromator and downstream towards the sample, Soller collimators are placed in the neutron beam, as well as between the sample and the neutron detector. Together with the so-called take-off angle of the neutrons reflected from the monochromator, the three collimators define the instrumental resolution of a $\mathrm{CW}$ diffractometer (Caglioti et al., 1958). PEARL is based at the $2 \mathrm{MW}$ research reactor of TU Delft (van Eijck et al., 2016). Despite the relatively low brilliance of this neutron source, the diffractometer can be considered a rather competitive medium-resolution instrument owing to its high take-off angle at the monochromator and the lack of collimators. The measuring time for typical samples ranges from $10 \mathrm{~min}$ to a day depending on the sample (crystal symmetry, neutron scattering cross section, amount of sample etc.). The instrument was conceived to operate at standard wavelengths of 2.51, 1.67 and $1.33 \AA$, but because of its placement on a radial beam tube that looks directly towards the reactor core, shorter-wavelength under-moderated neutrons are available at a reasonable flux loss compared with the standard settings. The shorter wavelengths allow us to collect the diffraction pattern up to $Q=13 \AA^{-1}$. As such, besides the typical powder diffraction used to study crystals that exhibit long-range order, PEARL can be used to perform PDF analysis of samples that lack such long-range order. Short-range order can be found in amorphous systems, in nanostructures, and, for instance, in the catalytically active sites of metal-organic frameworks (MOFs) and zeolites. To extract such short-range order from a high- $Q$ diffraction pattern, a PDF analysis is required.

\subsection{The PDFgetN3 program}

$P D F g e t N 3$ is released bundled in a Python package diffpy . pdfgetx, which can be obtained from http://www.diffpy.org. An updated version of the PDFget $X 3$ tool for X-rays (Juhás et al., 2013) is also included. The data conversion in PDFgetN3 acts as a chain of simple transformations that take a pair of input vectors $\left(x_{\text {in }}, y_{\text {in }}\right)$ and return transformed values $\left(x_{\text {out }}\right.$, $\left.y_{\text {out }}\right)$. The transformations can be listed from the PDFgetN3 interactive prompt using the pdfgetter.describe() function and their respective intermediate outputs are available in the pdfgetter.results variable. The chain of transformations is configurable and can be extended with userdefined operations. The PDFgetN3 and PDFget X3 programs have two pre-defined configuration modes, 'neutron' and 'xray', which set up transformations suitable for processing neutron or X-ray powder data. The essential difference between the modes is in using constant neutron scattering lengths versus $Q$-dependent X-ray scattering factors for the intensity normalization in equation (5). The configuration modes can be selected by passing the --mode option to the program and thus running the pdfgetn3 program is equivalent to executing pafgetx3--mode=neutron. Besides the neutron mode, the new release of PDFgetN3/ $P D F g e t X 3$ provides an additional parameter, two thetazero, to correct for goniometer offset in the scattering data. The parameter specifies the actual zero angle in diffractometer degrees and has proved crucial for obtaining high-quality PDFs from the PEARL data. Another major development in the new software is the added support for Python 3 (versions 3.4 or later) with a compatibility to Python 2.7 preserved. The software release also includes numerous bug fixes to 
PDFget $X 3$ and required updates for the developments of third-party libraries.

Because neutron scattering lengths are independent of $Q$, PDFgetN3 displays some specific behaviors that are different from the X-ray case. First, the obtained PDF is independent of the chemical composition used in data processing. Different chemical formulas result in a constant PDF re-scaling proportional to $\left\langle b_{i}^{2}\right\rangle /\left\langle b_{i}\right\rangle^{2}$, but otherwise the shape of the obtained curves is identical. PDFgetN3 also shows a similar rescaling behavior after subtraction of a constant background $B$ from the input raw intensities. The overall PDF scale changes as

$$
C(B)=\frac{\left\langle I_{\mathrm{m}}\right\rangle-B}{\left[\left\langle I_{\mathrm{m}}\right\rangle-B\right]^{2}+\left[\operatorname{std}\left(I_{\mathrm{m}}\right)\right]^{2}},
$$

where $\operatorname{std}\left(I_{\mathrm{m}}\right)$ is the standard deviation of all raw intensities and $\left\langle I_{\mathrm{m}}\right\rangle$ their average. The maximum PDF scale is then attained at

$$
B=B_{\max }=\left\langle I_{\mathrm{m}}\right\rangle-\operatorname{std}\left(I_{\mathrm{m}}\right) .
$$

This is because background-corrected intensities are rescaled to the order of $\left\langle b_{i}\right\rangle^{2}$ before passing to equation (5), which changes the output signal scale, while the constant offset is completely filtered away by the polynomial correction for the structure-independent signal (Juhás et al., 2013).

\section{Experimental}

The procedure was tested with samples of nickel and a NIST standard sapphire $\left(\alpha-\mathrm{Al}_{2} \mathrm{O}_{3}\right.$, corundum $)$ recorded at the PEARL diffractometer at room temperature using the 755 reflection in the germanium monochromator, resulting in a constant neutron wavelength of $1.098 \AA$. Data were collected over a $2 \theta$ range of $10.58-158.98^{\circ}$ with a pixel angular width of approximately $0.105^{\circ}$. This corresponds to $Q$ values ranging from 1.056 to $11.253 \AA^{-1}$. The data were collected in $21 \mathrm{~h}$ for sapphire and $80 \mathrm{~min}$ for nickel. Instrumental background and scattering intensities from the vanadium sample holder were measured separately.

$\mathrm{X}$-ray measurements for comparison were carried out at the XPD (28-ID-2) powder diffractometer at the National Synchrotron Light Source II (NSLSII), Brookhaven National Laboratory. The sapphire powder was placed in a $0.7 \mathrm{~mm}$-thick glass capillary and mounted perpendicular to the monochromatic X-ray beam of $0.2370 \AA$ wavelength. The scattering intensities were collected at room temperature using a PerkinElmer flat-panel aSi detector positioned $207 \mathrm{~mm}$ behind the sample. The exact detector distance and orientation were calibrated using the FIT2D (Hammersley et al., 1996; Hammersley, 2016)

Figure 1 refinement.
Table 1

Rietveld (GSAS-II) analysis results for the PEARL neutron powder diffraction data recorded at a wavelength of $1.098 \AA$.

\begin{tabular}{lll}
\hline & Nickel & Sapphire \\
\hline$a(\AA)$ & 3.5244 & $4.7594 \dagger$ \\
$c(\AA)$ & - & $12.9923 \dagger$ \\
$U_{\text {iso }}\left(\AA^{2}\right)$ & 0.00476 & $0.00279(\mathrm{Al}), 0.00327(\mathrm{O})$ \\
$Z$ ero correction $\left(^{\circ}\right)$ & -0.1742 & -0.158 \\
$R_{\mathrm{w}}$ & 0.046 & 0.075 \\
\hline
\end{tabular}

$\dagger$ Fixed value from NIST certificate.

program, which was also utilized to integrate pixel counts into a one-dimensional intensity versus $Q$ powder pattern. Finally, the data were corrected for empty capillary background and transformed to PDFs with the PDFget X3 software (Juhás et al., 2013).

The neutron diffraction patterns were analyzed by the Rietveld method using the GSAS-II program (Toby \& Von Dreele, 2013). The neutron PDFs (and the comparison $\mathrm{X}$-ray PDFs) were further analyzed by PDF structure refinements using the SrFit package embedded in the DiffPy-CMI software (Juhás et al., 2015). This allowed for automated iterative refinement of the relevant structure parameters as well as the zero offset of the diffractometer angle and the neutron wavelength (at a fixed unit cell).

\section{Results}

\subsection{Rietveld refinement of the diffraction patterns}

The empty can and vanadium sample holder background were removed prior to Rietveld analysis. An additional background was fitted using a simple three- or five-parameter Chebyshev polynomial. Instrumental line broadening profiles were determined using a sapphire NIST standard. The diffraction patterns for nickel and sapphire are displayed in Fig. 1 together with the respective Rietveld refinements.

The refined parameters can be found in Table 1 . The refined cell constants for nickel are in good agreement with values
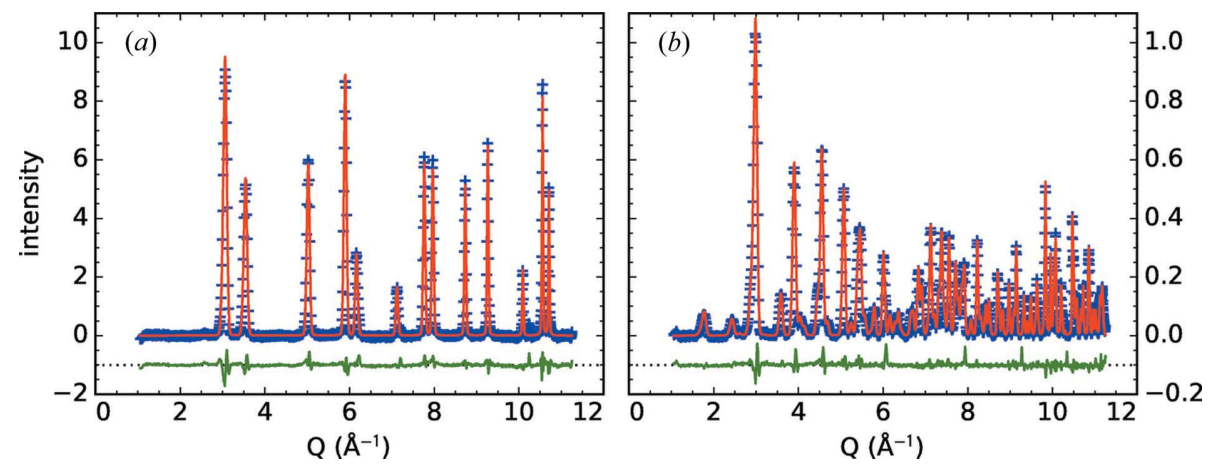

Rietveld refinements of neutron diffraction patterns from $(a)$ nickel and $(b)$ sapphire. The red line is the calculated and blue crosses the measured curves. Offset below, in green, is the difference curve. For both materials the instrumental and empty can background were removed prior to Rietveld 

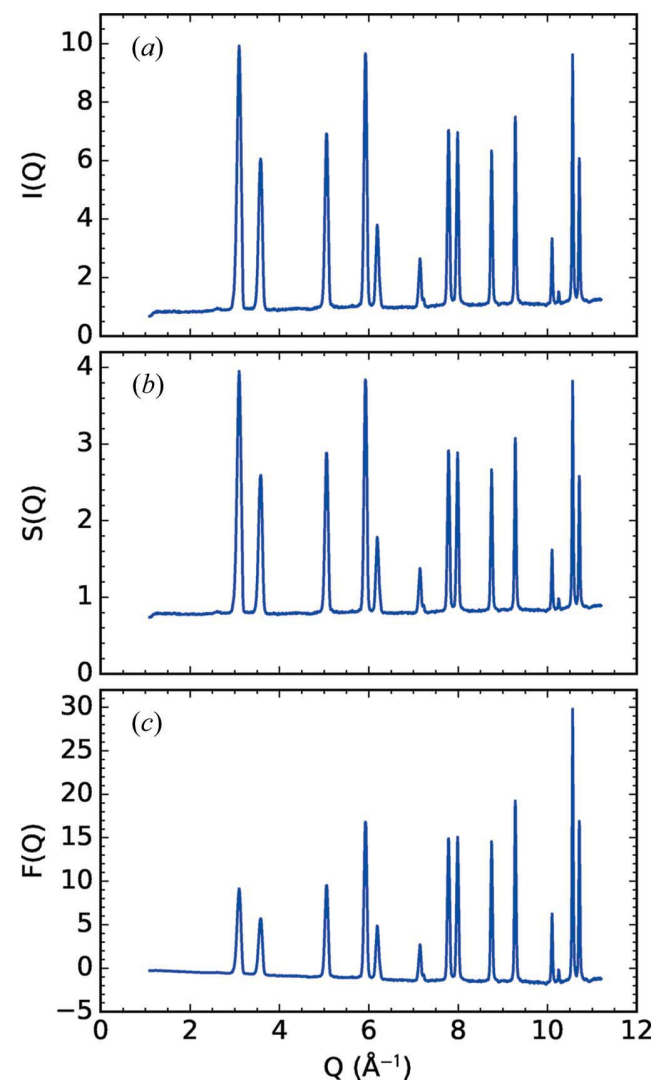

Figure 2

The intermediate results of PDF extraction from the nickel powder pattern measured at PEARL with monochromator setting 755. (a) The measured intensity $I(Q),(b)$ the structure function $S(Q)$ after scaling and polynomial corrections, and $(c)$ the corresponding reduced structure function $F(Q)$.

reported by Wyckoff (1963) and Suh et al. (1988). The refinement of the sapphire standard was conducted with cell parameters fixed according to its NIST certificate and with refinable neutron wavelength, instrumental zero, atom positions and isotropic displacement parameters. The obtained results are in good agreement with literature values (Kirfel \& Eichhorn, 1990; Lewis et al., 1982; Ishizawa et al., 1980; Newnham \& de Haan, 1962; Lutterotti \& Scardi, 1990).

\subsection{PDF extraction}

The background-corrected (i.e. instrumental and vanadium sample holder background removed) scattering profile for the nickel and sapphire samples was analyzed with PDFgetN3. The results of the various corrections contributing to the $F(Q)$ and $S(Q)$ functions for the nickel sample are presented in Fig. 2.

\subsection{PDF analysis}

The PDFs for nickel and sapphire were refined using the SrFit module in

Figure 3
Table 2

Comparison of PDF analyses for nickel.

\begin{tabular}{|c|c|c|c|}
\hline & $\begin{array}{l}\text { PDFget } X 3 \\
\text { Fig. } 4\end{array}$ & $\begin{array}{l}\text { PDFgetN3 (755) } \\
\text { Fig. } 3(a)\end{array}$ & $\begin{array}{l}\text { PDFgetN3 (955) } \\
\text { Fig. } 3(b)\end{array}$ \\
\hline$\lambda(\AA)$ & 0.14277 & 1.0989 (fixed) & $0.95539(16)$ \\
\hline$Q_{\max }\left(\AA^{-1}\right)$ & 26 & 11.2 & 12.9 \\
\hline Zero correction $\left(^{\circ}\right)$ & - & $-0.391(15)$ & $-0.447(17)$ \\
\hline$a$ & $3.5238(8)$ & $3.5180(6)$ & 3.5180 (fixed) \\
\hline$Q_{\text {damp }}\left(\AA^{-1}\right)$ & $0.056(2)$ & $0.037(2)$ & $0.041(3)$ \\
\hline$\delta_{2}$ & $2.5(2)$ & $1.3(6)$ & $3.5(7)$ \\
\hline$U_{\text {iso }}\left(\AA^{2}\right)$ & $0.005727(92)$ & 0.00437 (19) & $0.00391(20)$ \\
\hline$R_{\mathrm{w}}$ & 0.087 & 0.051 & 0.074 \\
\hline
\end{tabular}

the DiffPy-CMI framework (see Fig. 3 and Fig. 5). This allowed for iterative refinement of a number of parameters, including process parameters (zero offset) and structural parameters (scale factor, $Q$-resolution damping factor, displacement parameters and unit-cell parameters), at the same time. The fitted zero correction compensated for minor imperfections in the monochromator crystal alignments and in sample centering. The PDF extraction does not yet include error propagation. The standard errors of the fitted variables were therefore evaluated by repeating PDF fits at Nyquist sampling $\left(\Delta r=\pi / Q_{\max }\right)$, which minimizes error correlations between data points and allows the estimation of PDF errors from the difference between measured and fitted curves (Farrow et al., 2011; Bevington et al., 1992). In order to extend the $Q$ range we also recorded the scattering pattern for nickel using the 955 reflection of the monochromator, which translates to a constant wavelength of $0.955 \AA$. This increased the $Q_{\text {max }}$ from 11.2 to $12.9 \AA^{-1}$ and improved the $r$ resolution in the PDF as displayed in Fig. 3(b). The refinement of the 955 data was conducted with a variable wavelength and with the cell parameter fixed to the outcome of the 755 fit. This refinement gave a neutron wavelength of 0.9554 (2) $\AA$, which is in excellent agreement with an independent calibration from a Rietveld fit of the sapphire standard $(\lambda=0.95507 \AA)$ and attests to the consistency and accuracy of the results at both monochromator settings. Table 2 presents a full summary
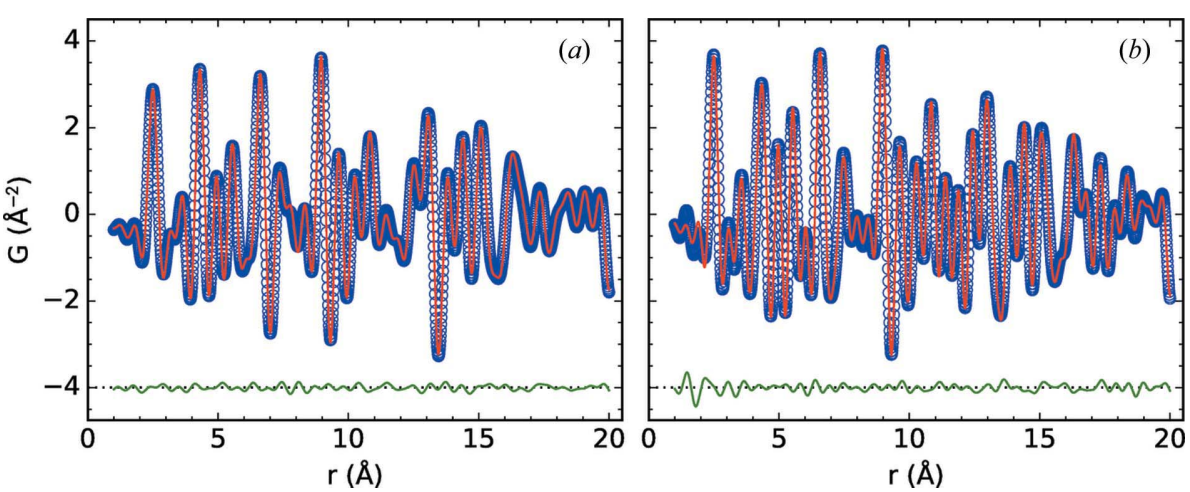

Refinement of nickel PDFs measured at the PEARL instrument using $(a)$ monochromator reflection $755\left(\lambda=1.098 \AA, Q_{\max }=11.2 \AA^{-1}, R_{\mathrm{w}}=0.051\right)$ and $(b)$ monochromator reflection 955 . The second fit optimized the wavelength for a fixed unit cell obtained from the first fit and yielded $(\lambda=$ 0.95539 (16) $\left.\AA, Q_{\max }=12.9 \AA^{-1}, R_{\mathrm{w}}=0.074\right)$. Experimental PDFs are plotted as blue circles and fitted curves as red lines, and the difference is offset below in green. 
Table 3

Comparison of PDF analyses for sapphire.

\begin{tabular}{lll}
\hline & $\begin{array}{l}\text { PDFget X3 (NSLS-II) } \\
\text { Fig. 5 }(a)\end{array}$ & $\begin{array}{l}\text { PDFgetN3 (755) } \\
\text { Fig. 5 }(b)\end{array}$ \\
\hline$\lambda(\AA)$ & 0.2370 & 1.0989 \\
$Q_{\max }\left(\AA^{-1}\right)$ & 23.5 & 11.2 \\
Zero correction $\left({ }^{\circ}\right)$ & - & $-0.377(15)$ \\
$a(\AA)$ & $4.7572(11)$ & $4.7519(12)$ \\
$c(\AA)$ & $12.9855(42)$ & $12.9731(28)$ \\
$z(\mathrm{Al})$ & $0.35236(26)$ & $0.35221(37)$ \\
$x(\mathrm{O})$ & $0.30581(93)$ & $0.30664(45)$ \\
$Q_{\text {damp }}\left(\AA^{-1}\right)$ & $0.01(2)$ & $0.031(3)$ \\
$\delta_{2}$ & $0.6(5)$ & - \\
$U_{\text {iso }}(\mathrm{Al})\left(\AA^{2}\right)$ & $0.00438(36)$ & $0.00157(55)$ \\
$U_{\text {iso }}(\mathrm{O})\left(\AA^{2}\right)$ & $0.00724(68)$ & $0.00361(35)$ \\
$R_{\mathrm{w}}$ & 0.164 & 0.071 \\
\hline
\end{tabular}

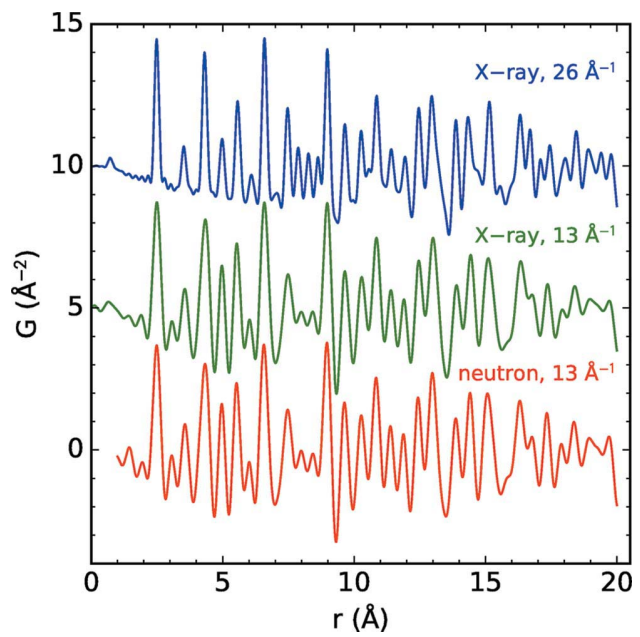

Figure 4

Comparison of $\mathrm{Ni}$ PDF obtained from constant-wavelength neutron scattering using the 955 monochromator setting (bottom red line) with the synchrotron X-ray PDF from Juhás et al. (2013) (top blue line), where the $Q$ range extended to $Q_{\max }=26 \AA^{-1}$. The middle green line is the X-ray PDF processed at the same $Q_{\max }=12.9 \AA^{-1}$ as the neutron scattering data. The PDF curves were extracted with PDFgetN3 and $P D F g e t X 3$, respectively. of nickel fit results together with a fit to the synchrotron X-ray data from Juhás et al. (2013).

The unit-cell sizes found for nickel with neutron diffraction are slightly smaller $(0.2 \%)$ than the values found above in the Rietveld refinement (Table 1), and are also smaller by a similar amount than that found in the PDF analysis of the synchrotron-derived data. Nevertheless, all values lie well within the range of 3.516-3.524 $\AA$ reported in the literature for nickel in cubic close-packed structure at ambient temperature (Wyckoff, 1963; Owen \& Yates, 1936; Jette \& Foote, 1935; Lundqvist, 1947; Suh et al., 1988). The agreement between the $\mathrm{X}$-ray and neutron PDFs is very good when they are processed with the same $Q_{\max }$, as can be seen by comparing the bottom two curves in Fig. 4 . The X-ray data processed with a higher $Q_{\max }$ are shown in the top curve for comparison. There is a significant loss of real-space resolution due to the limited $Q$ range in the neutron measurement, which results in broader peaks in the neutron data. The shorter $Q$ range also results in larger-amplitude termination ripples. This makes it hazardous to attribute meaning to individual peaks in the PDF, though it does not bias modeling which includes termination effects. As an example, a clear peak is evident at $r=3 \AA$, but there is no pair of atoms in the structure at that distance. These issues clearly improve when the data are collected at the 955 monochromator setting, and at either setting the data can be accurately modeled, as evidenced by the excellent match of the refined PDFs (Fig. 3). In general the peak amplitudes in X-ray and neutron PDFs differ because of the different scattering factors. However, this occurs only when peaks are formed by different atomic species, and for single-element materials such as nickel the PDF amplitudes from both techniques are equal.

The results from the model fits to the sapphire data are presented in Fig. 5 and Table 3. The refined values deviate only slightly from the parameters found by Rietveld analysis (Table 1) and from the X-ray PDF data. The unit-cell axes found in the PDF analysis are again $0.2 \%$ shorter than those found with Rietveld refinement, and slightly below the range found in the literature (4.754-4.7605 $\AA$ for $a$, and 12.9877$12.9956 \AA$ for $c$ ) (Kirfel \& Eichhorn, 1990; Lewis et al., 1982; Ishizawa et al., 1980; Newnham \& de Haan, 1962; Lutterotti \& Scardi, 1990). This deviation appears inconsequential for the overall structure as the refined fractional coordinates $z$ $(\mathrm{Al})$ and $x(\mathrm{O})$ agree within their error estimates with the literature values (Newnham \& de Haan, 1962). The X-ray PDF refinement of sapphire utilized data with $Q_{\max }$ about twice as large as the neutron case; however, it yielded a noticeably larger difference (Fig. 5) and a higher fit residual than the neutrons $\left(R_{\mathrm{w}}=0.164\right.$ versus 0.071$)$. The lower quality of the X-ray fit may be caused by valence deviations in the bonded $\mathrm{Al}-\mathrm{O}$ sites, which affect their

Refinement of sapphire PDFs measured by (a) NSLS-II synchrotron X-ray scattering at wavelength $0.2370 \AA, Q_{\max }=23.5 \AA^{-1}$, and $(b) \mathrm{CW}$ neutron scattering using monochromator reflection 755 , wavelength $1.098 \AA$ А,$Q_{\max }=11.2 \AA^{-1}$. 
actual scattering factors and the resulting PDF amplitudes. We find that the effect of valence on fits to these X-ray PDFs is not small. The reported $R_{\mathrm{w}}=0.164$ was obtained using scattering factors for neutral $\mathrm{Al}$ and $\mathrm{O}$ sites at valences 13 and 8 . A trial refinement with ionic $\mathrm{Al}^{3+}, \mathrm{O}^{2-}$ (valences 10,10) actually gave a much worse fit $\left(R_{\mathrm{w}}=0.37\right)$, revealing the high sensitivity of the PDF model to $\mathrm{Al}-\mathrm{O}$ bonding and effective valences. It is beyond the scope of this paper to refine the valences to see whether this could improve the fit.

Finally, we note that we also used PDFgetN3 to take intensity data from a time-of-flight neutron experiment that had been reduced to $I(Q)$ but not normalized or Fourier transformed, and it gave a comparable quality PDF to one calculated using all the corrections.

\section{Conclusions}

We have presented a new software program, PDFgetN3, for the extraction of pair distribution functions from powder neutron diffraction intensities. The comparison between results from Rietveld refinement and PDF analysis, as well as the comparison between neutron PDF analysis and X-ray PDF analysis, shows good agreement. The ad hoc correction method introduced in PDFget $X 3$ is therefore also applicable in the PDF extraction with PDFgetN3 presented here. The software is available for download at http://www.diffpy.org.

\section{Acknowledgements}

The authors thank Dr Milinda Abeykoon for essential help with synchrotron X-ray experiments.

\section{Funding information}

These measurements were conducted at the beamline 28-ID-2 of the National Synchrotron Light Source II, a US Department of Energy (DOE) Office of Science User Facility operated for the DOE Office of Science by Brookhaven National Laboratory under contract No. DE-SC0012704. PJ was supported by the New York State BNL Big Data Science Capital Project under the US Department of Energy contract No. DE-SC-00112704. SJLB was supported by the US Department of Energy, Office of Science, Office of Basic Energy Sciences (DOE-BES) under contract No. DESC00112704. JNL and ETCV acknowledge support by the Albemarle Catalysts Company BV. ETCV was also funded by the NWO (Netherlands Organization for Scientific Research) project TKINCI.2015.005.

\section{References}

Bevington, P. R., Robinson, D. K. \& Bunce, G. (1992). Data Reduction and Error Analysis for the Physical Sciences, 2nd ed. New York: McGraw-Hill.

Billinge, S. J. L. (2010). Physics, 3, 25.

Billinge, S. J. L. \& Farrow, C. L. (2013). J. Phys. Condens. Matter, 25, 454202.

Caglioti, G., Paoletti, A. \& Ricci, F. P. (1958). Nucl. Instrum. 3, 223 228.

Egami, T. \& Billinge, S. J. L. (2012). Underneath the Bragg Peaks: Structural Analysis of Complex Materials, 2nd ed. Amsterdam: Elsevier.

Eijck, L. van, Cussen, L. D., Sykora, G. J., Schooneveld, E. M., Rhodes, N. J., van Well, A. A. \& Pappas, C. (2016). J. Appl. Cryst. 49, 1398-1401.

Farrow, C. L., Shaw, M., Kim, H.-J., Juhás, P. \& Billinge, S. J. L. (2011). Phys. Rev. B, 84, 134105.

Fischer, H. (2016). SofqSoft: Software For Treating S(q) Neutron Scattering Data from Disordered Systems, https://forge.epn-campus. eu/projects/sofqsoft.

Hammersley, A. P. (2016). J. Appl. Cryst. 49, 646-652.

Hammersley, A. P., Svensson, S. O., Hanfland, M., Fitch, A. N. \& Hausermann, D. (1996). High. Pressure Res. 14, 235-248.

Howe, M. A. \& McGreevy, R. L. Z. P. (1996). CORRECT: A Correction Program for Neutron Diffraction Data. Technical Report, The Studsvik Neutron Research Laboratory, Uppsala University, Sweden.

Ishizawa, N., Miyata, T., Minato, I., Marumo, F. \& Iwai, S. (1980). Acta Cryst. B36, 228-230.

Jette, E. \& Foote, F. (1935). J. Chem. Phys. 3, 605-616.

Juhás, P., Davis, T., Farrow, C. L. \& Billinge, S. J. L. (2013). J. Appl. Cryst. 46, 560-566.

Juhás, P., Farrow, C., Yang, X., Knox, K. \& Billinge, S. (2015). Acta Cryst. A71, 562-568.

Kirfel, A. \& Eichhorn, K. (1990). Acta Cryst. A46, 271-284.

Lewis, J., Schwarzenbach, D. \& Flack, H. D. (1982). Acta Cryst. A38, 733-739.

Lundqvist, D. (1947). Ark. Mineral. Och Geol. 24, 1-12.

Lutterotti, L. \& Scardi, P. (1990). J. Appl. Cryst. 23, 246-252.

Newnham, R. E. \& de Haan, Y. M. (1962). Z. Kristallogr. 117, 235237.

Owen, E. \& Yates, E. (1936). London Edinb. Dubl. Philos. Mag. J. Sci. 21, 809-819.

Peterson, P. F., Gutmann, M., Proffen, Th. \& Billinge, S. J. L. (2000). J. Appl. Cryst. 33, 1192.

Petkov, V. (1989). J. Appl. Cryst. 22, 387-389.

Qiu, X., Thompson, J. W. \& Billinge, S. J. L. (2004). J. Appl. Cryst. 37, 678.

Soper, A. K. \& Barney, E. R. (2011). J. Appl. Cryst. 44, 714-726.

Suh, I.-K., Ohta, H. \& Waseda, Y. (1988). J. Mater. Sci. 23, 757-760.

Toby, B. H. \& Von Dreele, R. B. (2013). J. Appl. Cryst. 46, 544-549.

Wyckoff, R. W. G. (1963). Crystal Structures, Vol. 1, 2nd ed. New York: Wiley. 